\title{
Mechanism of Pipe End Deformation after Cutting of Square Steel Pipe Formed by Roll Forming*
}

\author{
Takuo Nagamachi ${ }^{1}$, Takefumi Nakako ${ }^{2}$ and Daisuke Nakamura ${ }^{2}$ \\ ${ }^{1}$ Institute of Technology and Science, University of Tokushima, Tokushima 770-8506, Japan \\ ${ }^{2}$ Steel \& Technology Development Laboratories, Nisshin Steel Co., Ltd., Sakai 592-8332, Japan
}

\begin{abstract}
Square steel pipes are reshaped from welded round pipes by roll-forming. Pipe end deformation after cutting of the square steel pipe was investigated by experimentation and by three-dimensional finite element simulation. Each direction of the residual stresses composed of longitudinal stress and longitudinal-peripheral shear stress becomes reverse with respect to the outer and inner surfaces. A large opening deformation arises at the back end of the product by the release of those residual stresses. The side part receives bending deformation in the surrounding area of the contact zone with a roll. The residual stresses comprising longitudinal stress and longitudinal-peripheral shear stress increase as a result of the reverse bending deformation downstream of the roll center position. The absolute value of residual stress decreases with decreased initial wall thickness. [doi:10.2320/matertrans.P-M2013822]
\end{abstract}

(Received September 18, 2012; Accepted July 9, 2013; Published September 6, 2013)

Keywords: roll forming, square steel pipe, pipe end deformation, residual stress, finite element (FE) simulation

\section{Introduction}

When a pipe produced by cold roll forming is cut into lengths, deformation of the cut section occurs under the release of residual stresses. This deformation is called as pipe end deformation. A large pipe end deformation produces products being out of the standard. And they make welding operation difficult. Development of a roll forming method that may prevent pipe end deformation is desired because straightening brings about poor production efficiency.

Many papers have been published with respect to pipe end deformation. Kato et al. ${ }^{1)}$ measured dimensions in cut sections of channel steel to clarify the tendency of closing deformation of front end and opening deformation of back end. In addition, they examined the relation between mechanical properties of the sheet metal and pipe end deformation. Mihara et al. ${ }^{2}$ ) conducted experiments on forming of U-shaped rib, having found that inserting inner rolls of small diameter in U-shaped cross-section at the last stand was effective to suppress end deformation. Conducting a series of forming experiments on the channel, hat and Cchannel steels, Ona et al. ${ }^{3)}$ described that residual bending moment and residual torsional moment were the cause of pipe end deformation. Itami et l. $^{4)}$ conducted FE simulation of sizing process in circular pipe and described that longitudinal residual stress that is not uniform in a peripheral direction was the factor of pipe end deformation. For a square pipe, Itadani et al. ${ }^{5)}$ examined dimensions of the cut section and found opening deformation for both front end and back end. Some studies are conducted in this way but the relation between bending deformation and residual stress in rollformed pipe are yet uncertain. Additionally, sufficient information related to the mechanism by which released residual stress cause the pipe end deformation remains unclear.

The authors ${ }^{6)}$ investigated the relation between cross sectional shapes of roll-formed square pipe and forming

*This Paper was Originally Published in Japanese in J. JSTP 52 (2011) 1083-1087. conditions. By focusing on pipe end deformation in square pipes, the deformation is examined by measuring the cut section shape. Then, FE simulation of forming and cutting processes were conducted to find residual stress in the pipe and to discuss the mechanism of the pipe end deformation.

\section{Experiment and Analysis Method}

Figure 1 presents a schematic view of the forming process used in this study. The ram of an oil pressure cylinder pushes the circular pipe into square-shaped roll holes made by four roll sets of Nos. 1, 2 and 3, and square steel pipe is discharged from the last roll set. In this process, no rolls are driven. Rolls and their dimensions are presented in Fig. 2. Table 1 summarizes the mechanical property of circular pipe material derived from tensile tests. The circular pipe material is a ferritic stainless steel. Generally, pipe end deformation is important in final product that had passed successive roll sets of Nos. 1, 2 and 3. In addition to the final product, we measured the geometry of the cut section after just the No. 1 roll set and that cut after successive roll sets Nos. 1 and 2 . The cutting is performed by using a wire-cut electric discharge machine (EDM) and the shape of a section $1 \mathrm{~mm}$ distant from the end is measured using a $3 \mathrm{D}$ coordinate measuring instrument.

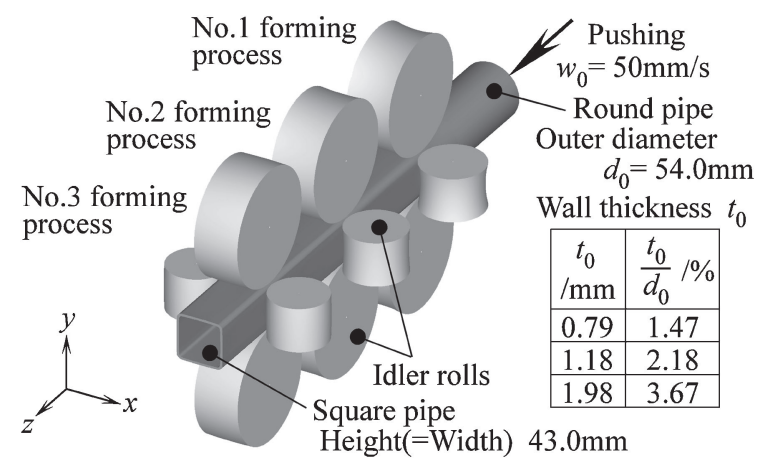

Fig. 1 Schematic illustration of forming processes. 


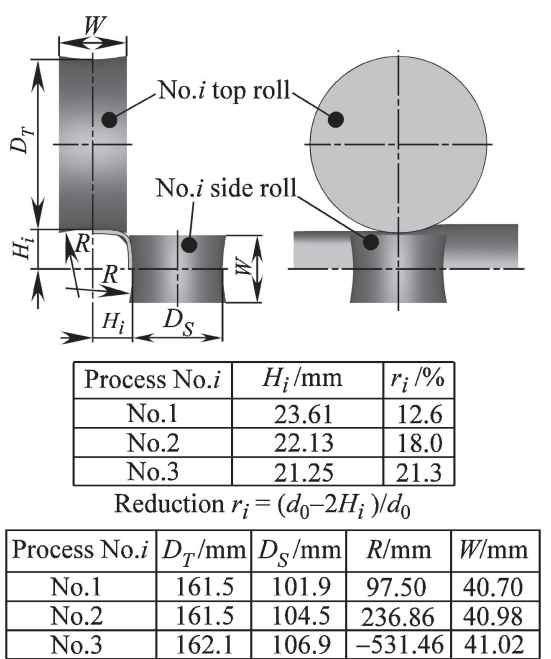

Fig. 2 Notations and dimensions of rolls.

Table 1 Mechanical properties of round pipe.

\begin{tabular}{lccc}
\hline Wall thickness $t_{0} / \mathrm{mm}$ & 0.79 & 1.18 & 1.98 \\
Young's modulus/GPa & & 193.2 & \\
Poisson's ratio & & 0.3 & \\
Yield stress/MPa & 395 & 428 & 465 \\
$n$ value & 0.226 & 0.204 & 0.179 \\
Tensile strength/MPa & 477.3 & 481.4 & 505.2 \\
\hline
\end{tabular}

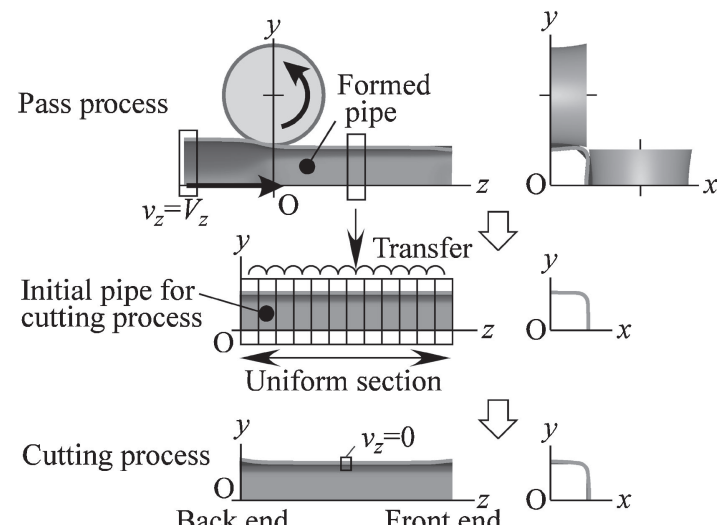

$v_{z}$ : Boundary nodal velocity in $z$ direction

Fig. 3 Procedure of calculation for cutting process.

FE simulation was conducted using a static implicit scheme applied to transient elasto-plastic analysis. First, forming simulations for each of roll sets Nos. 1, 2 and 3 were conducted separately using the procedure described in Ref. 6). Then, cutting processes were simulated using the process demonstrated in Fig. 3. Process are as follows. (1) Select one section in the stationary deformation region in the forming process; (2) Transfer coordinates and stressstrain data in a longitudinal direction to generate a model that has uniform sectional shape in the longitudinal direction; (3) Remove boundary condition at both ends to allow deformation at the cutting section; (4) Determine the pipe end deformation by displacement of nodes that produce a force imbalance. Numerous elements in the thickness direction are necessary to calculate the deformation of elastic recovery. As

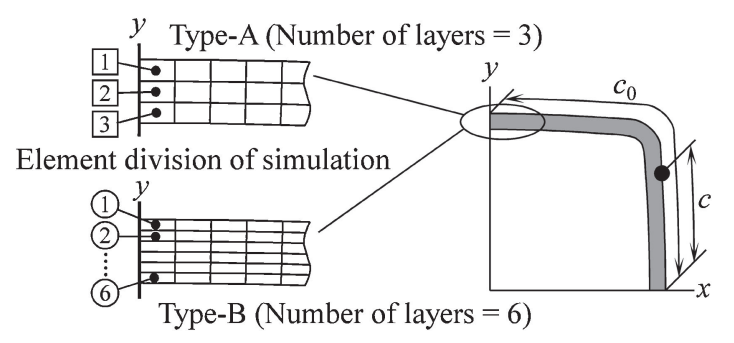

Fig. 4 Schematic illustration of elements in thickness direction.

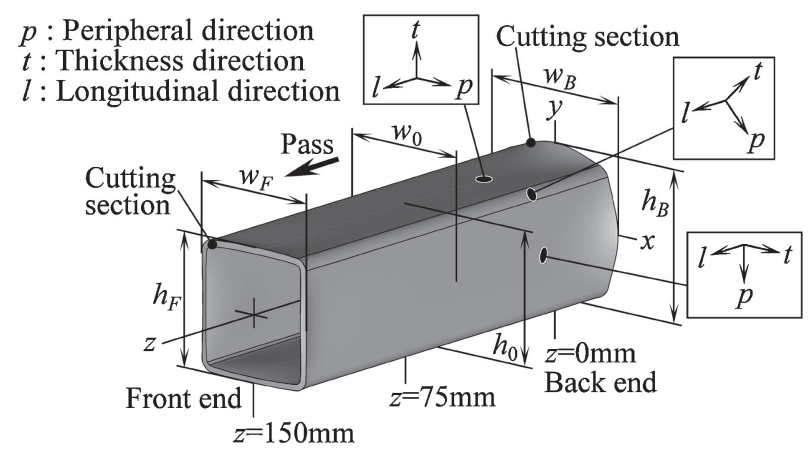

Fig. 5 Notations of representing shape of cut pipe.

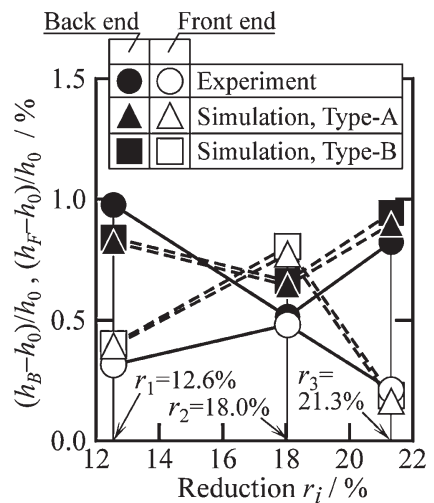

(a) Height

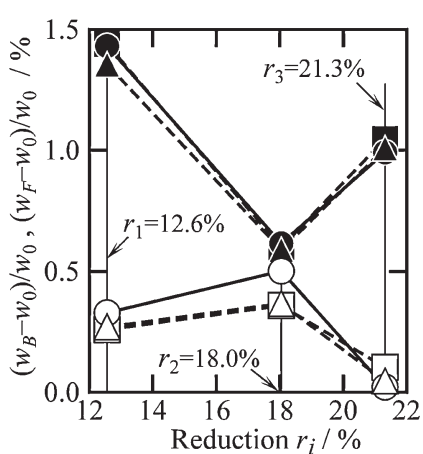

(b) Width
Fig. 6 Increasing rate of height and width in cutting section of pipe, $r_{1}=12.6 \%$ : pipe formed by No. 1 rolls, $r_{2}=18.0 \%$ : pipe formed by No. 1 and No. 2 rolls, $r_{3}=21.3 \%$ : product, $t_{0} / d_{0}=3.67 \%$.

shown in Fig. 4, 2 types of cutting layer were compared. The Type-A has three layers in the thickness direction and the Type-B has six layers. The analysis domain for FE analysis was cut by hexahedral elements with eight nodes. A generalpurpose code of DEFORM-3D Ver. 10.1 was used to perform calculations.

\section{Results and Discussion}

3.1 Pipe end deformation and its generation mechanism

The notation to representing shape of cut pipe is shown in Fig. 5. In this part, the residual stress and pipe end deformation that occur in pipe with $t_{0} / d_{0}=3.67 \%$, where $t_{0}=$ wall thickness and $d_{0}=$ round pipe outer diameter (refers Fig. 1) were discussed. Figures 6(a) and 6(b) respectively show increasing rates of height and width in the cutting section. Open symbol $(\bigcirc, \square, \triangle)$ stand for the rate of increase in the front end (downstream cut section) and 


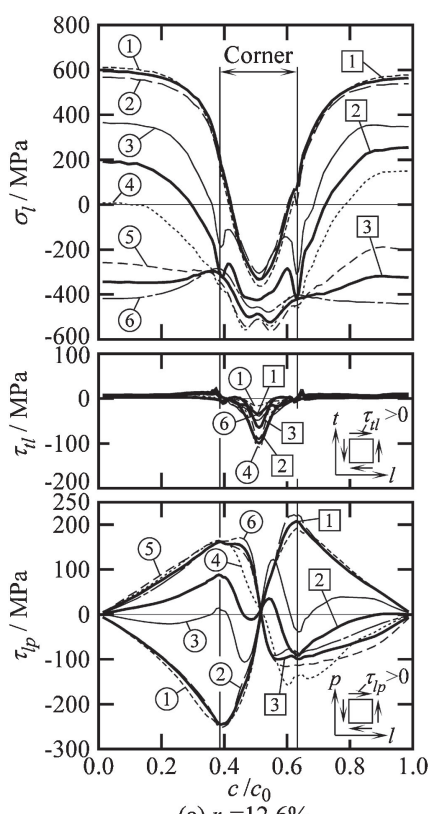

(a) $r_{1}=12.6 \%$

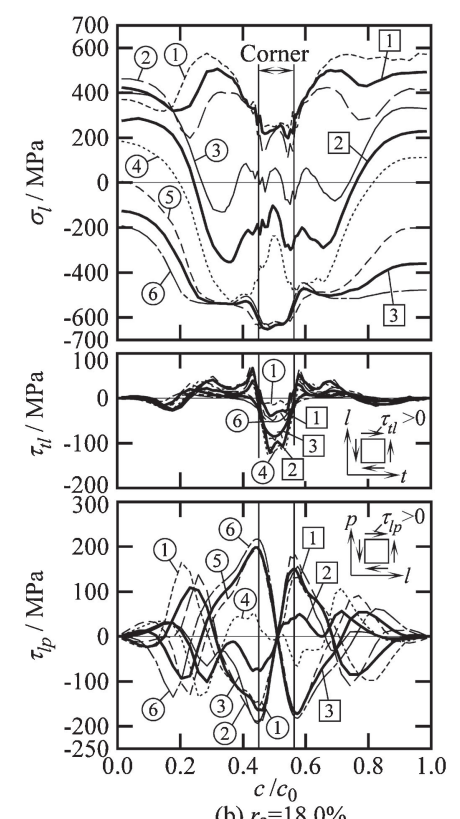

(b) $r_{2}=18.0 \%$

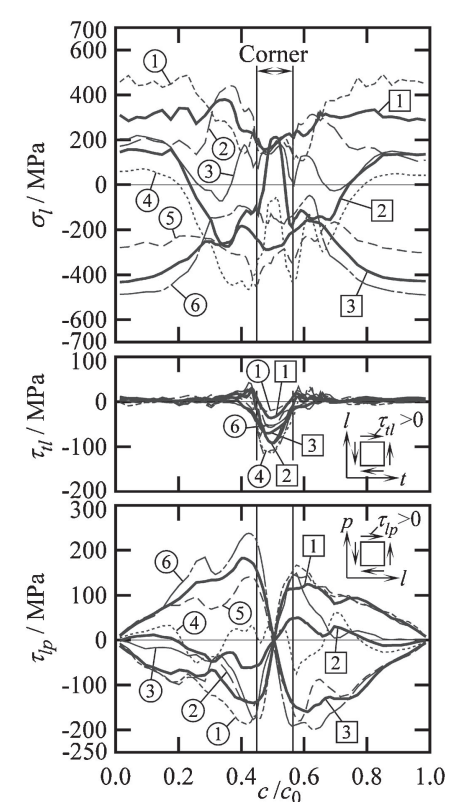

(c) $r_{3}=21.3 \%$

Fig. 7 Peripheral distribution of residual stress ( $\sigma_{1}$ : longitudinal stress, $\tau_{\mathrm{t} l}$ : shear stress in thickness-longitudinal direction, $\tau_{\mathrm{lp}}$ : shear stress in longitudinal-peripheral direction) of pipe, (a) $r_{1}=12.6 \%$ : pipe formed by No. 1 rolls, (b) $r_{2}=18.0 \%$ : pipe formed by No. 1 and No. 2 rolls, (c) $r_{3}=21.3 \%$ : product, $1-3$ : Type-A, (1)-6): Type-B, $t_{0} / d_{0}=3.67 \%$, simulation results.

solid symbol $(\boldsymbol{\bullet}, \boldsymbol{\square}, \mathbf{\Delta})$ stand for the rate of increase in the back end (upstream cut section). The horizontal axis shows reduction $r_{i}$ (see Fig. 2), where $r_{1}=12.6 \%, r_{2}=18.0 \%$ and $r_{3}=21.3 \%$ respectively which were formed by No. 1 forming, No. 2 forming and No. 3 forming rolls. All measured values are positive, which indicates opening deformation. In increasing rates for $r_{1}=12.6 \%$ and $r_{3}=$ $21.3 \%$, back end values are larger than those of front end both in height and width, suggesting larger opening deformation at the back end. Increasing rates derived from simulation show a similar increasing rate with those of measured values. FE simulation with six layers in the thickness direction (Type-B) provided almost same results with the three layers (Type-A). It is believed that the occurrence of pipe end deformation of product formed by roll forming is caused by shear stresses in thickness-longitudinal direction and longitudinal-peripheral direction. ${ }^{7)}$ However no research seems to have explained in detail the generation of longitudinal-peripheral shear deformation and the mechanism by which that deformation causes pipe end deformation. Therefore, in this research, residual shear stresses and longitudinal residual stress are discussed. Notations to represent shape of cut pipe are shown in Fig. 5, which the peripheral direction as $p$-direction, thickness direction as $t$-direction, longitudinal direction as $l$-direction. Peripheral distribution of longitudinal stress $\sigma_{1}$, thickness-longitudinal shear stress $\tau_{\mathrm{tl}}$ and longitudinal-peripheral shear stress $\tau_{\mathrm{lp}}$, at the steady part of pipe simulated are portrayed respectively in Fig. 7(a) for $r_{1}=12.6 \%$, (b) for $r_{2}=18.0 \%$ and (c) for $r_{3}=21.3 \%$. Horizontal axis, $c / c_{0}$ represents the peripheral position (see Fig. 4), where $c / c_{0}=0$ center of lateral side, $c / c_{0}=1$ center of top side and $c / c_{0}=0.5$ center of the corner. Numbers by symbol of $\square$ and $\bigcirc$ in the graph represent Type-A and Type-B simulation results respectively. (1), 1 represent outermost layers and (6), 3 innermost layers. In this research, the values of thickness-longitudinal shear stress $\tau_{\mathrm{tl}}$ are almost zero, because of the thin circular pipes used. So, we conclude that thickness-longitudinal shear stress $\tau_{\mathrm{tl}}$, does not affect pipe end deformation. Therefore, longitudinal stress $\sigma_{\mathrm{l}}$, and longitudinal-peripheral shear stress $\tau_{\mathrm{lp}}$ are discussed in this section.

In order to discuss this issue, first, we discuss about pipe formed by No. 1 rolls set with $r_{1}=12.6 \%$. As shown in Fig. 7(a), for longitudinal stress $\sigma_{\mathrm{l}}$, the occurrence of large tensile residual stress at the outermost layer and large compressive residual stress at the innermost layer can be seen. Further details of the stress can be seen in Fig. 8(a), which is a schematic representation of longitudinal stress $\sigma_{1}$, when $\sigma_{1}>0$ at outermost layer and $\sigma_{1}<0$ at the innermost layer. When such a pipe with residual $\sigma_{1}$ is cut, the outermost layer will shrink in to the longitudinal direction and the innermost layer will elongate in to the longitudinal direction. Displacement vectors in these movements are shown by arrows in the cutting section of Fig. 8(a). As depicted in the schematic drawing of Fig. 9(a), both of front and back ends deform in opening direction by released $\sigma_{1}$. Whereas, in the inner plane residual shear stress $\tau_{\mathrm{lp}}$ shown in Fig. 7(a), that of the outermost layer has the opposite sign (positive or negative) with that of the innermost layer. Outermost layer with $\tau_{\mathrm{lp}}>0$ and innermost layer with $\tau_{\mathrm{lp}}<0$ are shown schematically in Fig. 8(b). Here we assume no deformation at the corner part constrained by stiffness. Then, the release of residual $\tau_{\mathrm{lp}}$ by cutting the pipe makes the outermost layer of side parts displace from the back end to front end direction and the innermost layer of side parts in opposite direction. Displacement vectors in these movements are shown by arrows in the cutting section of Fig. 8(b). As shown in the schematic drawing of Fig. 9(b), the front end deforms in the closing direction and back end in opening direction by released $\tau_{\mathrm{lp}}$. From above, we could fine that the superposition 


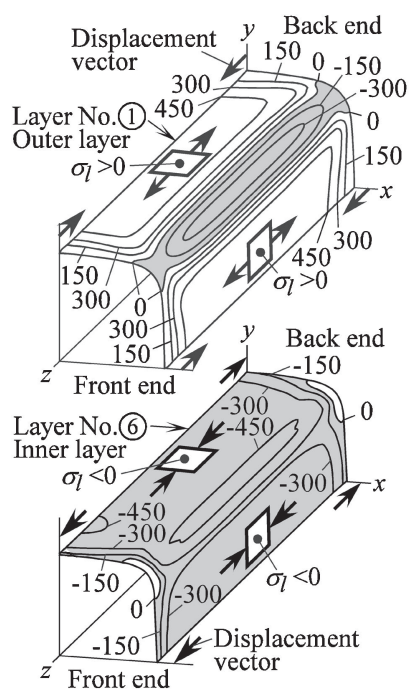

(a) $\sigma_{l} / \mathrm{MPa}$

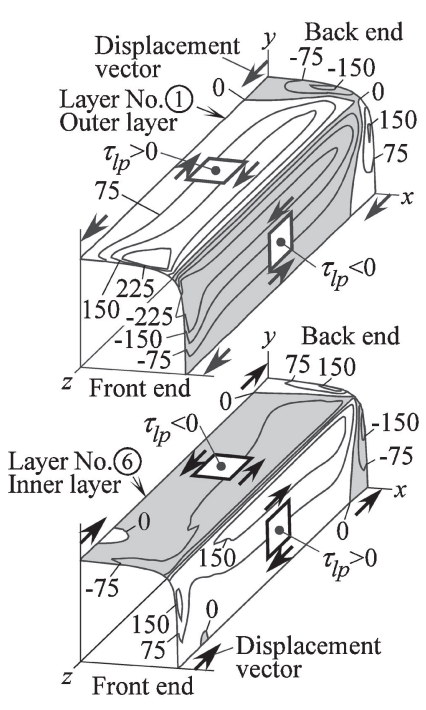

(b) $\tau_{l p} / \mathrm{MPa}$
Fig. 8 Illustration of residual stress $\left(\sigma_{1}\right.$ : longitudinal stress, $\tau_{\mathrm{lp}}$ : shear stress in longitudinal-peripheral direction) in outer and inner layer of pipe formed by No. 1 rolls and displacement vector at pipe end, $t_{0} / d_{0}=3.67 \%$, simulation results, Type-B.

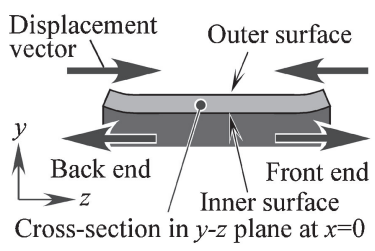

(a) Relief of $\sigma_{l}$

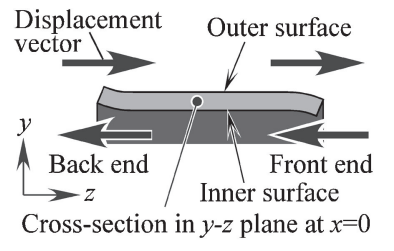

(b) Relief of $\tau_{l p}$
Fig. 9 Illustration of pipe end deformation occurred by relief of residual stress. of opening deformation by released $\sigma_{1}$ and that by released $\tau_{1 \mathrm{p}}$ brings about large opening deformation at the back end. Also, we find that the relation between residual stresses $\sigma_{1}$ and $\tau_{\mathrm{lp}}$ and pipe end deformation in Fig. 7(c) resembles with Fig. 7(a).

Then, we proceed to discussion of the No. 2 forming process, with $r_{2}=18.0 \%$. Taking notice of $\tau_{\mathrm{lp}}$ of the outermost layer (1) and innermost layer (6) by Type-B simulation portrayed in Fig. 7(b), when $c / c_{0}=0.2, \tau_{\mathrm{lp}}>0$ for (1) and $\tau_{\mathrm{lp}}<0$ for (6) and when $c / c_{0}=0.4, \tau_{\mathrm{lp}}<0$ for (1) and $\tau_{\mathrm{lp}}>0$ for (6). Longitudinal-peripheral residual shear stresses with opposite direction of outermost and innermost layers might bring about pipe end deformation shown in Fig. 9(b). However, two deformations exist in opposite directions at the same side part of the cut section, cancelling each other out, suppressing deformation and reducing the effect of $\tau_{\mathrm{lp}}$. Therefore, for $r_{2}=18.0 \%$ we might expect similar magnitude of front end and back end deformation mainly by the effect of $\sigma_{1}$, as shown in Fig. 6 .

\subsection{Generation mechanism of residual stress}

Residual stresses $\sigma_{1}$ and $\tau_{1 \mathrm{p}}$ at outermost and innermost layers might be strongly depend on contact state of a pipe with a roll and on the deformation state of the pipe. In this session, first, the generation of residual stress using the contact region with No. 1 rolls and distribution of strain rate in the pipe with $t_{0} / d_{0}=3.67 \%$ are discussed. Figures 10 (a) and 10 (b) portray the distribution of the longitudinal strain rate, $\dot{\varepsilon}_{1}$ and shear strain rate in longitudinal-peripheral direction, $\dot{\gamma}_{\text {lp }}$ in a pipe being formed by No. 1 rolls. These $\dot{\varepsilon}_{1}$ and $\dot{\gamma}_{1 \mathrm{p}}$ are plastic strain rates. In the figure, dotted marks "๑"s show points of contact with rolls and dash lines show $z=0$ of the longitudinal coordinate at the roll center. Both $\dot{\varepsilon}_{1}$

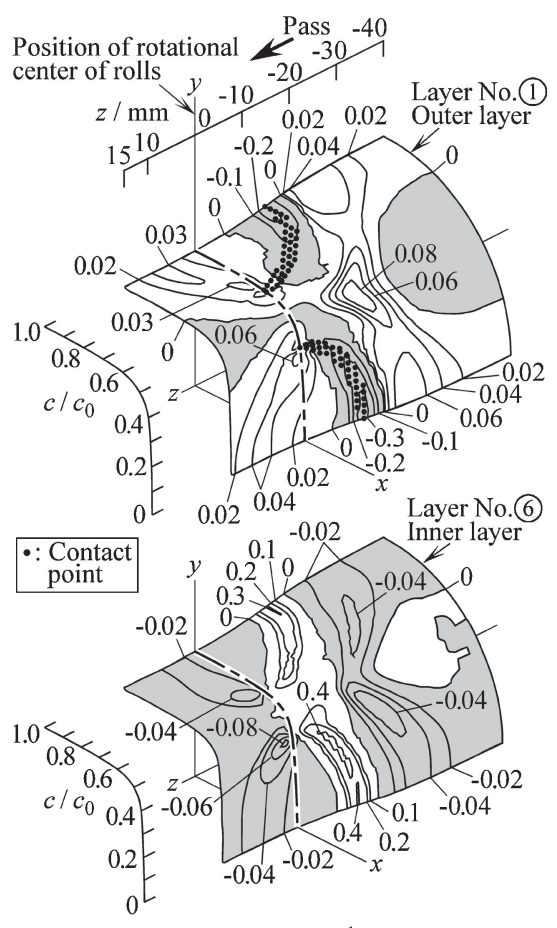

(a) $\dot{\varepsilon}_{l} / \mathrm{s}^{-1}$

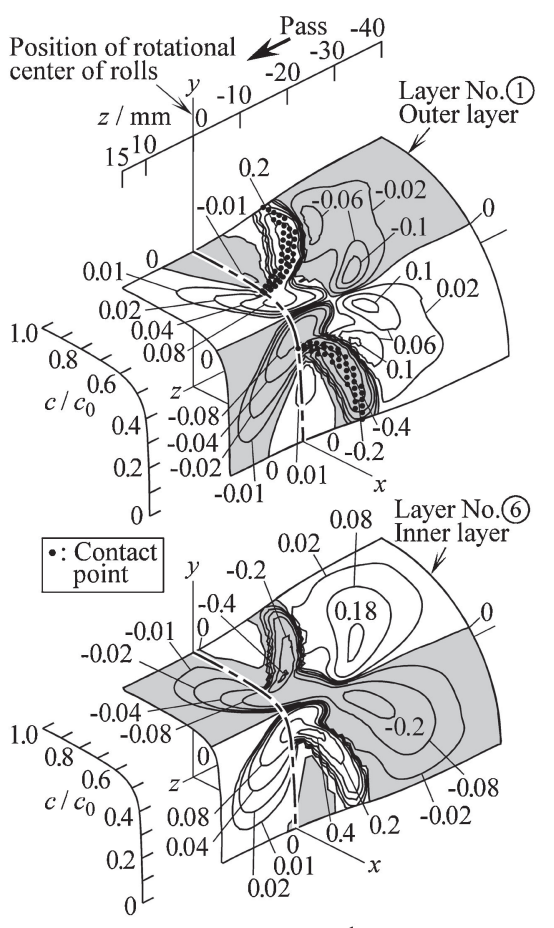

(b) $\dot{\gamma}_{l p} / \mathrm{s}^{-1}$

Fig. 10 Distribution of strain rate $\left(\dot{\varepsilon}_{1}\right.$ : longitudinal strain rate, $\dot{\gamma}_{l p}$ : shear strain rate in longitudinal-peripheral direction) in outer and inner layer of pipe being formed by No. 1 rolls, $t_{0} / d_{0}=3.67 \%$, simulation results, Type-B. 
and $\dot{\gamma}_{l p}$ have large absolute values at the contact region with the rolls, where large deformation velocity prevails. However, in at $z=10 \mathrm{~mm},\left|\dot{\varepsilon}_{1}\right|>0$ and $\left|\dot{\gamma}_{1 \mathrm{p}}\right|>0$ have a small absolute value, which means small plastic deformation downstream of the roll center. At the central region of side $\left(c / c_{0}=0\right.$ or $\left.c / c_{0}=1\right)$ in Fig. 10(a), $\dot{\varepsilon}_{1}>0$ at outermost layer and $\dot{\varepsilon}_{1}<0$ at innermost layer. In Fig. 10(b) at the side region where $c / c_{0}<0.5$ and $z>10 \mathrm{~mm}, \dot{\gamma}_{\mathrm{lp}}<0$ at the outermost layer and $\dot{\gamma}_{\mathrm{lp}}>0$ at the innermost layer. Whereas, at the side region in the same figure, where $c / c_{0}>0.5$ and $z>10 \mathrm{~mm}$, $\dot{\gamma}_{\mathrm{lp}}>0$ at the outermost layer and $\dot{\gamma}_{\mathrm{lp}}<0$ at the innermost layer. Peripheral distributions of these $\dot{\varepsilon}_{1}$ and $\dot{\gamma}_{\mathrm{lp}}$ coincide, in terms of sign (positive or negative), with the sign distribution of $\sigma_{1}$ and $\tau_{\text {lp }}$ portrayed in Fig. 7(a). As conclusion, we could find that residual stresses occur in the direction in which plastic deformation takes place when $(z=10 \mathrm{~mm})$ from the roll center.

We can estimate the deformation of pipe being formed by No. 1 rolls by observing the signs of $\dot{\varepsilon}_{1}$ and $\dot{\gamma}_{\text {lp }}$ presented in Fig. 10 and by preparing a schematic drawing as shown in Fig. 11. Bending deformation at the central region of top side A-B-C might be described as follows, keeping up the pipe stream. The pipe is first bent convexly in upstream region A. Then it is bent concavely by roll contact at the $\mathrm{B}$ region. This is explained clearly by the longitudinal distribution of curvature shown in Fig. 7 of our previous report. ${ }^{6)}$ Because the longitudinal curvature is zero in the downstream region $\mathrm{C}$, the central region of top side bent concavely by roll contact at the $\mathrm{B}$ region is bent reversely to straighten in the downstream region. At this instant, strain rate $\dot{\varepsilon}_{1}$ tensile outside and compressive inside occurs and longitudinal residual stress $\sigma_{1}$ tensile outside and compressive inside are generated simultaneously.

Furthermore, $\dot{\gamma}_{\mathrm{lp}}$ occurs at a region D close to the corner as presented in Fig. 11, and it has opposite sign between outside and inside. This is explained as described below. In region $\mathrm{C}$, such a longitudinal reverse bending deformation occurs that is tensile outside and compressive inside. Furthermore, the corner part marked by $\mathrm{E}$ experiences a peripheral bending deformation that is tensile outside and compressive inside. Altogether, a resulting deformation surrounding the contact region is tensile outside and compressive inside in the radial direction from center of the contact region. Because the radial direction in the region $\mathrm{D}$ coincides neither with the longitudinal direction nor with the peripheral direction, deformation in the region $\mathrm{D}$ that is tensile outside and compressive outside develops as longitudinal-peripheral shear strain that results in $\dot{\gamma}_{\mathrm{lp}}>0$ outside and $\dot{\gamma}_{\mathrm{lp}}<0$ inside. Simultaneously, longitudinal-peripheral shear stress appears and it results in residual shear stress such that $\tau_{\mathrm{lp}}>0$ outside and $\tau_{\mathrm{lp}}<0$ inside. These patterns of deformation were observed in pipe being formed by No. 3 rolls. ${ }^{8)}$

We next discuss the residual stress generation mechanism using the contact region with No. 2 rolls and distribution of

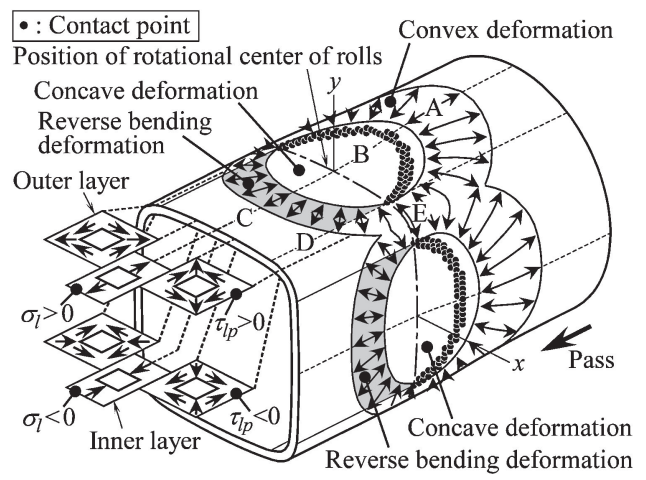

Fig. 11 Illustration of deformation of pipe being formed by No. 1 rolls and residual stress.

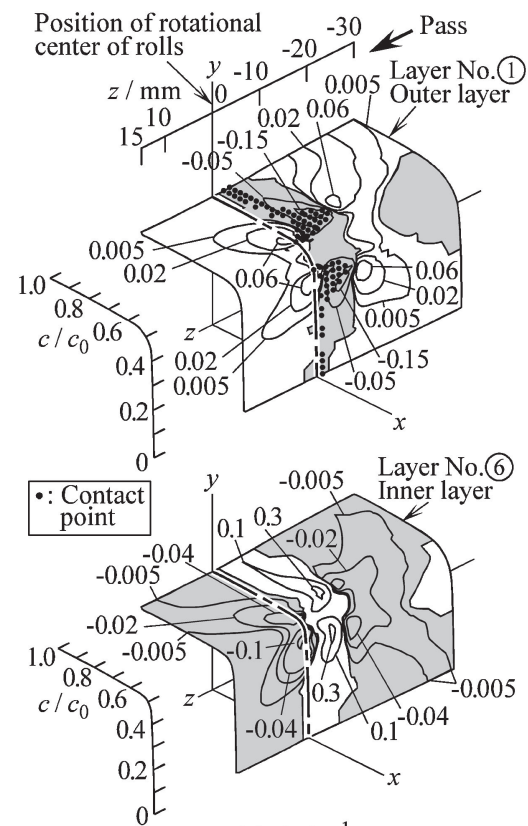

(a) $\dot{\varepsilon}_{l} / \mathrm{s}^{-1}$

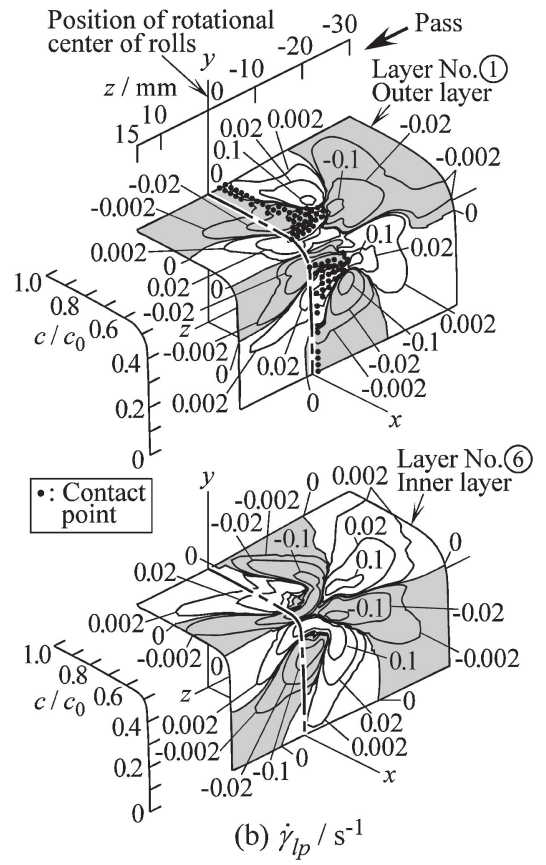

Fig. 12 Distribution of strain rate ( $\dot{\varepsilon}_{1}$ : longitudinal strain rate, $\dot{\gamma}_{l p}$ : shear strain rate in longitudinal-peripheral direction) in outer and inner layer of pipe being formed by No. 2 rolls, $t_{0} / d_{0}=3.67 \%$, simulation results, Type-B. 


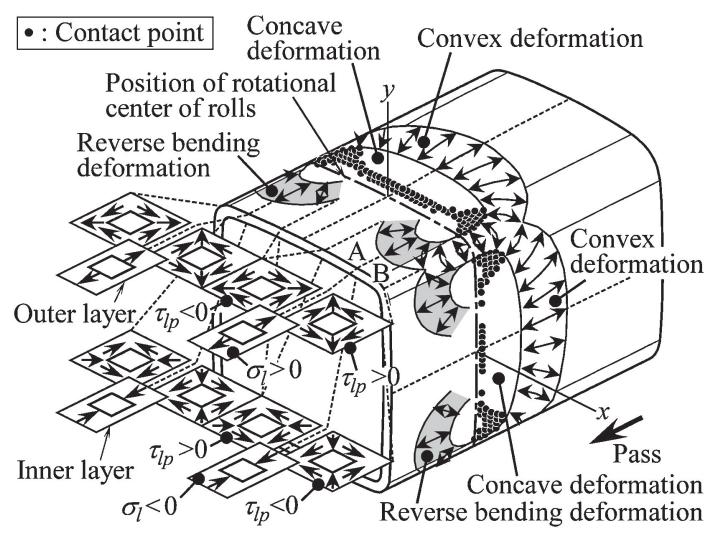

Fig. 13 Illustration of deformation of pipe being formed by No. 2 rolls and residual stress.

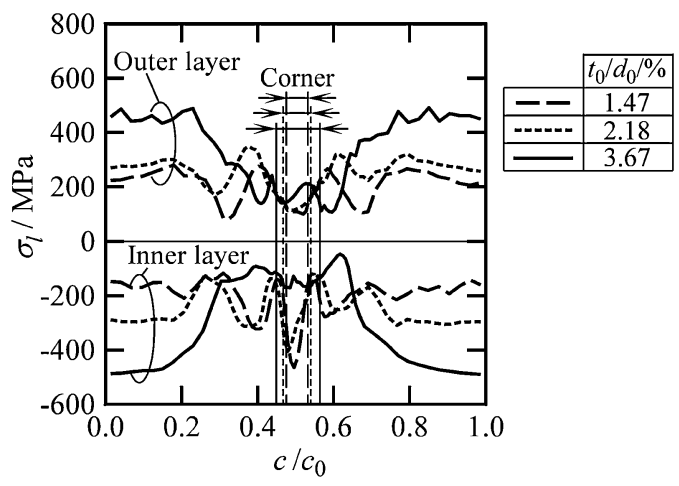

Fig. 14 Peripheral distribution of residual stress ( $\sigma_{1}$ : longitudinal stress) of product, simulation results.

strain rate in the pipe. In the side part with $z>0, \dot{\varepsilon}_{1}>0$ at outermost layer and $\dot{\varepsilon}_{1}<0$ at innermost layer as depicted in Fig. 12(a). For $z>10 \mathrm{~mm}$ in Fig. 12(b), $\dot{\gamma}_{\mathrm{lp}}>0$ at the outermost layer and $\dot{\gamma}_{\mathrm{lp}}<0$ at the innermost layer in the region around $c / c_{0}=0.2$. These signs of $\dot{\gamma}_{\text {lp }}$ are reversed completely in the region around $c / c_{0}=0.4$. Peripheral distributions of these $\dot{\varepsilon}_{1}$ and $\dot{\gamma}_{\text {lp }}$ coincide, in terms of sign, with the sign distribution of $\sigma_{1}$ and $\tau_{\mathrm{lp}}$ portrayed in Fig. 7(b). The contact region of No. 1 rolls with the pipe is one big semicircular shapes. However contact group of No. 2 rolls with pipe exists close to the corner. We can conduct image deformation surrounding the contact region as shown also in Fig. 11. It is tensile outside and compressive inside in the radial direction from center of the contact region. We can prepare a schematic drawing of deformation like that shown in Fig. 13. At point $\mathrm{A}$ in the figure $\tau_{\mathrm{lp}}$ is $\tau_{\mathrm{lp}}<0$ at the outermost layer and at point $B$ it is $\tau_{\mathrm{lp}}>0$ at the same layer. In summary, contact group which exists close to the corner brings about longitudinal-peripheral residual shear stresses in the same side with opposite signs.

\subsection{Relation between initial thickness of pipe and pipe end deformation}

The effect of the initial pipe thickness on longitudinal residual stress at outermost and innermost layers is presented

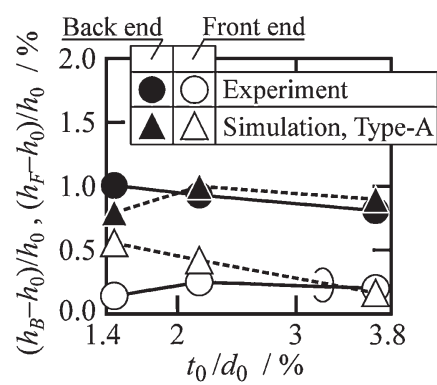

(a) Height

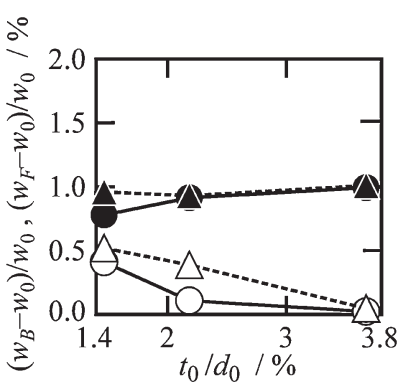

(b) Width
Fig. 15 Increasing rate of height and width in cutting section of product.

in Fig. 14. The thinner the initial thickness is, the smaller the absolute value of residual stress $\sigma_{1}$ is. However, deformation by elastic recovery appears to be greater than initial pipe thickness. As presented in Fig. 15, it is known that increasing the rate of dimension at cutting section is not definitely affected by the initial pipe thickness.

\section{Conclusion}

(1) Signs (positive or negative) of longitudinal residual stress and longitudinal-peripheral residual shear stress in sides of pipes are opposite in the outermost layer and innermost layer. Release of these residual stresses produces large end deformation at the back end (upstream end) of the pipe.

(2) Deformation surrounding the contact region is tensile outside and compressive inside in the radial direction from center of the contact region. Their deformation develops as longitudinal stress and longitudinalperipheral shear stress, and it is the origin of residual stress.

(3) The thinner the initial thickness is, the smaller the absolute value of residual stress $\sigma_{1}$ in the sides is. However, increasing the rate of dimension at the cutting section is not definitely affected by the thickness in this study.

\section{REFERENCES}

1) K. Kato, Y. Saito, Y. Nakawaki and H. Kanno: Proc. 28th Japanese Joint Conference for the Technology of Plasticity, (1977) pp. 357-359 (in Japanese).

2) Y. Mihara, T. Suzuki, T. Kamata and I. Yamanobe: NKK GIHO 86 (1980) 287-293 (in Japanese).

3) H. Ona, T. Jinba, J. Nakayama and S. Matsuda: J. JSTP 24 (1983) 434441 (in Japanese).

4) Y. Itami, M. Ataka and J. Shibata: J. JSTP 36 (1995) 1379-1384 (in Japanese).

5) M. Itadani and T. Toyooka: CAMP-ISIJ 10 (1997) 1121 (in Japanese).

6) T. Nagamachi, T. Nakako and D. Nakamura: Mater. Trans. 52 (2011) 2159-2164.

7) Japan Society for Technology of Plasticity (ed.): Roll Forming, (Corona Publishing, 1990) p. 20 (in Japanese).

8) T. Nagamachi, T. Nakako and D. Nakamura: Proc. 61st Japanese Joint Conference for the Technology of Plasticity, (2010) pp. 241-242 (in Japanese). 